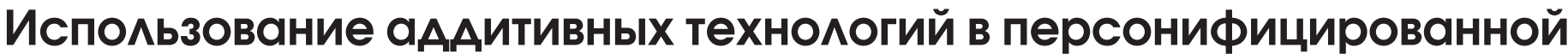 хирургии позвоночника (экспериментальное исслеАование)
}

\author{
И.А. Аоценко ${ }^{1}$ В.В. Котомцев², И.А. МеАвинский ${ }^{1,3}$, А.В. Савельев ${ }^{1}$, М.А. Ботов ${ }^{4}$ \\ тУНИИФ - филиал ФГБУ «НМИЦ ФПИ» Минздрава России, Екатеринбург, Россия \\ 2 ИИФ УрО РАН, Екатеринбург, РОссия \\ ЗФГОУ ВО УГМУ Минздрава России, Екатеринбург, Россия \\ ¿ФГАОУ ВО «УрФУ им. Первого президента России Б.Н. Ельцина», Екатеринбург, Россия
}

\begin{abstract}
РЕЗЮМЕ
Цель исследования: сравнение двух методов фиксациии позвоночно-двигательных сегментов у экспериментальных животных при переломовывихе одного из грудных позвонков.

Материал и методы: в основной группе (10 животных - собаки разных пород) применялась разрабатываемая технология фиксации позвоночно-двигательных сегментов персонифицированными титановыми пластинами с индивидуальным рельефом прилегающей к кости поверхности и встроенной системой навигациии в виде отверстий с предварительно рассчитанными углами наклона, в которые по принцииу минимально инвазивного проведения устанавливались винтовые фиксаторы. Пластины производились в дооперациионном периоде на $3 D$-принтере с применением аддитивных технологий и с учетом особенностей анатомии позвоночно-двигательного сегмента экспериментального животного на основе первичных данных, полученных из пакета DICOM при мультиспиральной компьютерной томографии пораженного сегмента позвоночника. Группу сравнения (контрольную) составили 10 собак с аналогичной травмой, у которых фиксация позвоночно-двигательных сегментов проводилась с помощью традиционной методики - транспедикулярными винтами.
\end{abstract}

Результаты исследования: группы сопоставимы по механизму и уровню (нижнегрудной отдел позвоночника) травмы с однотипными последствиями в виде переломовывиха одного из грудных позвонков, по возрасту и массе тела животных (возраст - 3,5 года, 95\% доверительный интервал [ДИ] 2,8-4,2; масса тела - 32,4 к2, 95\% ДИ 29,3-35,5). У всех животных из основной группы в послеоперациионном периоде получили положительный результат лечения в виде регресса неврологического дефицита при отсутствии значимой воспалительной послеоперацционной реакции. Применение данной технологии лечения позволило избежать перфорации позвоночного канала при установке винтовых фиксаторов и уменьшить интраоперациинную лучевую нагрузку на пациента и операциионную бригаду, а также увеличить стабильность фиксациии сегментов позвоночника и предотвратить переломы винтов. Заключение: нами была показана перспективность применения элементов разработанной технологии в хирургической практике лечения поражений позвоночно-двигательных сегментов различного генеза у человека.

Ключевые слова: аддитивные технологии, компьютерная томография, травмы позвоночника, доклинические исследования, минимально инвазивные вмешательства.

Для цитирования: Доцченко И.А., Котомцев В.В., Медвинский И.Д. и др. Использование аддитивных технологий в персонифицированной хирургии позвоночника (экспериментальное исследование). РМЖ. Медицинское обозрение. 2020;4(2):83-88. DOI: 10.32364/25876821-2020-4-2-83-88.

\section{Additive technologies in spinal personalized surgery (experimental study)}

\author{
I.A. Dotsenko1, V.V. Kotomtsev', I.D. Medvinskiy, ${ }^{1,3}$, A.V. Savel'ev', M.A. Botov ${ }^{4}$ \\ Ural Research Institute of Phthisiopulmonology — Branch of the National Medical Research \\ Center of Phthisiopulmonology and Infectious Diseases, Ekaterinburg, Russian Federation \\ IInstitute of Immunology and Physiology of the Ural Branch of the Russian Academy of \\ Sciences, Ekaterinburg, Russian Federation \\ ${ }^{3}$ Ural State Medical University, Ekaterinburg, Russian Federation \\ ${ }^{4}$ The First Russian President B.N. Yeltsin Ural Federal University, Ekaterinburg, Russian Federation
}

\begin{abstract}
Aim: to compare two techniques of the fixation of spinal motion segments in experimental animals with thoracic vertebral fracture/luxation. Patients and Methods: 10 animals (dogs) were included in the study group. These animals underwent spinal motion segment fixation by experimental technique using personalized titan plates with the individual shape of the surface adjacent to bone and integrated navigation system (apertures with pre-calculated tilt angle for minimally invasive screw fixation). Titan plates were manufactured preoperatively by a $3 D$ printer technique using additive technologies depending on the anatomy of spinal motion segment of the experimental animal based on the primary computed tomography DICOM imaging. 10 dogs with similar injury which underwent traditional surgery (spinal motion segment fixation using transpedicular screws) were included in the study group.

Results: the groups were matched by the mechanism and level (lower thoracic spine) of the injury with similar consequences (thoracic vertebral fracture/luxation), age (on average, 3.5 years, 95\% CI 2.8-4.2) and weight (on average, $32.4 \mathrm{~kg}$, 95\% CI 29.3-35.5) of experimental animals.
\end{abstract}


Postoperatively, the improvement of neurological deficiency and the lack of significant inflammation were reported in the study group. This technique prevents spinal canal perforation when placing screws, reduces intraoperative surgeon and patient radiation exposure, improves the stability of spinal segment fixation, and avoids screw fractures.

Conclusion: this novel technique is a promising surgical modality to manage human spinal motion segment damage of various origin.

Keywords: additive technologies, computed tomography, spinal injuries, preclinical studies, minimally invasive surgery.

For citation: Dotsenko I.A., Kotomtsev V.V., Medvinskiy I.D. et al. Additive technologies in spinal personalized surgery (experimental study). Russian Medical Review. 2020;4(2):84-88. DOI: 10.32364/2587-6821-2020-4-2-83-88.

\section{ВведЕНИЕ}

Стабилизация позвоночно-двигательных сегментов представляет определенные сложности, обусловленные особенностями биомеханики и анатомии пораженных и прилегающих к ним позвоночно-двигательных сегментов, что особенно актуально у пациентов со сформированной кифотической и/или сколиотической деформацией позвоночника на фоне спондилитов туберкулезной и неспецифической этиологии [1-9].

В настоящее время существует множество систем вентральной и дорзальной стабилизации позвоночно-двигательных сегментов, установка многих из них сопровождается травматизацией невральных структур [1-5, 7 , 8, 10-16]. Одним из решений данной проблемы может служить применение технологии накостного остеосинтеза с фиксацией соседних позвонков персонифицированными титановыми пластинами, имеющими индивидуальный рельеф прилегающей к кости поверхности и встроенную систему навигации в виде отверстий с предварительно рассчитанными углами наклона, в которые по принципу минимально инвазивного проведения устанавливались винтовые фиксаторы. Пластины производились путем прототипирования на 3D-принтере с применением аддитивных технологий и с учетом особенностей анатомии позвоночно-двигательного сегмента экспериментального животного на основе первичных данных, полученных из пакета DICOM при мультиспиральной компьютерной томографии (МСКТ) пораженного сегмента позвоночника [17-19]. Применение персонифицированных титановых пластин, во-первых, обеспечивает тесное прилегание к любым анатомическим ориентирам и учитывает особенности структуры костной поверхности позвоночника, во-вторых, позволяет вводить винтовые фиксаторы по заранее рассчитанной траектории и существенно снижать риски их мальпозиции с перфорацией позвоночного канала и травматизацией невральных структур. В настоящей статье представлен опыт применения разработанной нами технологии в эксперименте при травматических поражениях позвоночно-двигательных сегментов у собак.

Целью исследования является изучение возможности применения предлагаемой нами технологии фиксации позвоночно-двигательных сегментов с элементами навигации в эксперименте на животных.

\section{МАТЕРИАЛ И МЕТОДЫ}

Эксперимент получил одобрение этического комитета УНИИФ - филиала ФГБУ «Национальный медицинский исследовательский центр фтизиопульмонологии и инфекционных заболеваний» Минздрава России (протокол № 2 от 08.02.2018). Экспериментальное исследование было проведено на 20 животных (собаках разных пород) обоего пола, средний возраст - 3,5 года (95\% ДИ 2,8-4,2), средняя масса тела - 32,4 кг (95\% ДИ 29,3-35,5), с преимущественно схожим механизмом и последствиями травмы, приведшими к переломовывиху одного из грудных позвонков и грубому неврологическому дефициту по типу центрального парапареза с глубиной неврологических нарушений, оцененной по шкале Frankel [6] от А- до С-степени. По данной шкале степень A (F.A) - отсутствие двигательных и чувствительных функций ниже уровня травмы; степень В (F.B) - плегия, сохранена чувствительность или ее элементы; степень C (F.C) - выраженный парез (есть слабые движения), чувствительность сохранена; степень D (F.D) - слабый парез, чувствительность сохранена; степень E (F.E) - отсутствуют чувствительные или двигательные нарушения (независимо от рентгенологической картины повреждения позвонков).

Животные содержались в одинаковых условиях передержки (в индивидуальных клетках, на однотипном рационе), получали стандартную послеоперационную терапию и курс реабилитации. В зависимости от метода фиксации позвоночно-двигательных сегментов животные были разделены на две сопоставимые (условия содержания, рост и вес животных, механизм травмы, степень неврологического дефицита по шкале Frankel) группы по 10 особей в каждой.

В контрольной группе до операции после сбора анамнеза и обследования животного формировался первичный план хирургического лечения, в соответствии с которым после ламинэктомии позвонки фиксировали при помощи полиаксиальных транспедикулярных винтов, без использования какой-либо навигационной системы.

У животных из основной группы эксперимент проводился по следующему плану в соответствии с разработанной технологией:

1. Сбор анамнеза и первичное обследование животного, проведение обзорных спондилограмм в прямой и боковой проекциях. Показаниями для проведения МСКТ являлись клинические и рентгенологические признаки нестабильного перелома или переломовывиха грудных позвонков.

2. Первичное сканирование на МСКТ, формирование пакета данных в DICOM-формате. Обработка техническим специалистом и нейроортопедом полученного пакета данных DICOM и формирование первичного плана хирургического лечения.

3. Обработка пакета данных DICOM, создание первичной трехмерной модели поврежденного сегмента позвоночника с разделением на отдельную модель передней опорной колонны (включающую в себя тело, диски и ножки тела поврежденного позвонка) и заднюю опорную колонну (нижние и верхние суставные отростки, поперечные и остистый отростки поврежденного тела позвонка) [4-6]. 


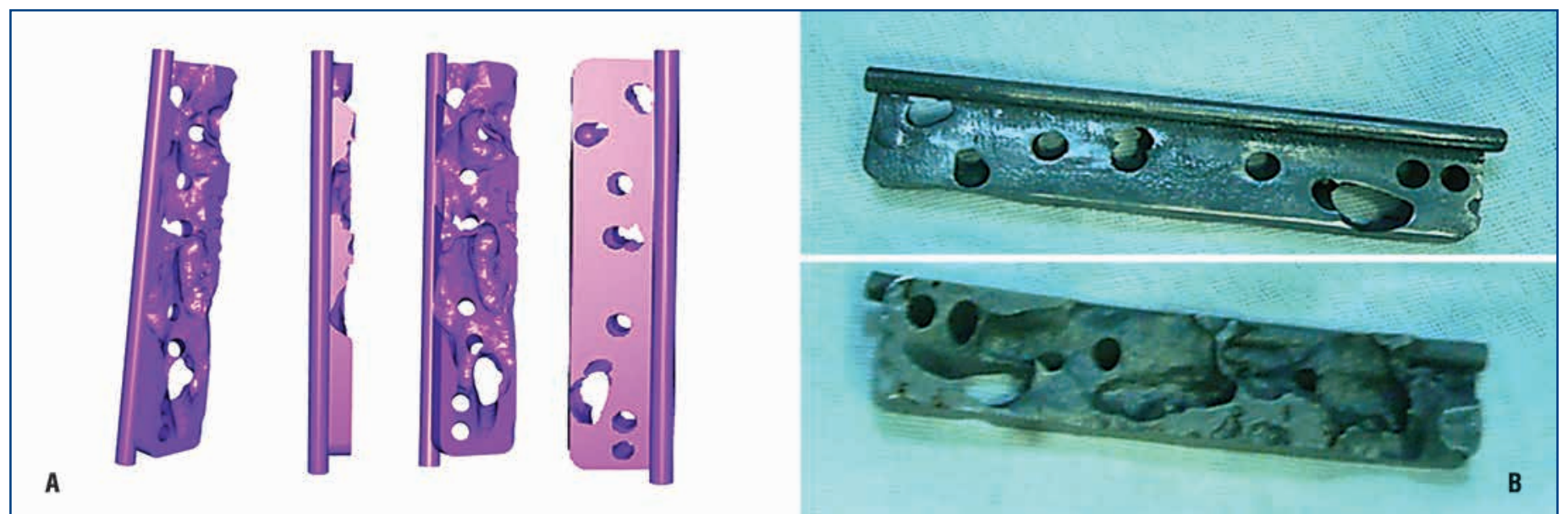

Рис. 1. А - вид пластины с лицевой и оборотной (прилегающей к кости) сторонами; В — пластина после печати на аддитивном принтере

Fig. 1. A - The front and the back (adjacent to bone) side of the plate; B - The plante printed on additive printer

4. Формирование анатомических файлов: восстановленная модель передней опорной колонны, модель задней опорной колонны, формирование модели позвоночника с восстановленным сагиттальным балансом. Утверждение плана хирургического лечения.

5. Формирование на 3D-модели проекта виртуально имплантированного в модель набора каналов винтовой фиксации [8] (рис. 1).

6. Сборка и формирование на основе построенных линий проведения винтов каналов-направителей и конечной персонифицированной модели пластины (рис. 2).

7. Печать персонифицированной пластины из пористого титана на 3D-принтере.

8. Стерилизация персонифицированной пластины и проведение хирургического вмешательства: задняя декомпрессия спинного мозга методом резекции дужки позвонков (ламинэктомия) с последующим скелетированием участка тел позвонков в месте установки пластины. На подготовленный участок накладывали персонифицированную пластину и фиксировали ее кортикальными винтами, после чего рану закрывали, используя рассасывающийся шовный материал. На кожу накладывали узловатый прерывистый шов из нерассасывающегося материала.

Исследование на предоперационном этапе включало: общий и биохимический анализы крови, рентгенографию и МСКТ позвоночного столба, оценку неврологического дефицита по шкале Frankel. После оперативного вмешательства все случаи мониторировались в динамике исследования в объеме общего и биохимического анализов крови, рентгенографии позвоночного столба, оценки неврологического дефицита по шкале Frankel в 1, 3, 10, 50 и 90-е сут после операции. МСКТ проводилась через 1, 3 и 6 мес. после хирургического вмешательства.

Статистический анализ проводился на базе программного обеспечения IBM SPSS Statistics 21.0. Количественные данные представлены в виде среднего и стандартного отклонения среднего $(\mathrm{M} \pm \mathrm{SD})$. С учетом небольшого объема совокупности для статистического анализа применяли методы непараметрической статистики. Сравнение результатов контрольной и основной групп проводили с использованием критерия Манна - Уитни для независи-

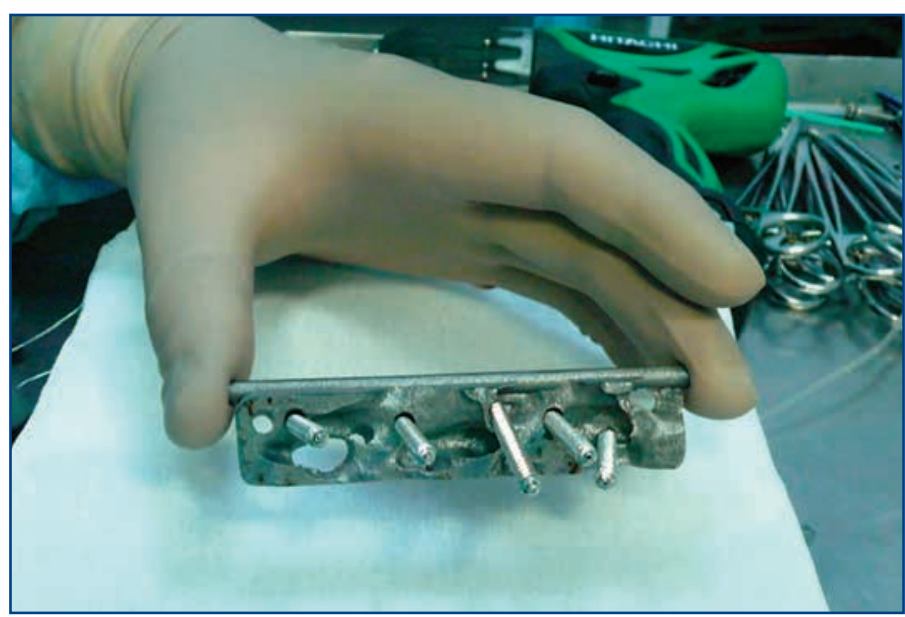

Рис. 2. Персонифицированная фриксирующая пластина и направления винтов

Fig. 2. Personalized fixating plate and screw orientation

мых наблюдений, т. к. распределение не соответствовало нормальному, а данные о степени неврологического дефицита на пред- и послеоперационном этапах для каждой из групп сравнивались с помощью критерия Вилкоксона для зависимых наблюдений. Уровень значимости был выбран $\alpha=0,05$.

\section{РЕЗУЛЬТАТЫ ИССЛЕДОВАНИЯ}

При сравнительном исследовании результатов общего и биохимического анализов крови животных в обеих группах на этапах мониторирования в послеоперационном периоде достоверных отличий не выявлено.

Рентгенограмма позвоночника животного из контрольной группы представлена на рисунке 3. По результатам рентгенографии и МСКТ позвоночного столба в послеоперационном периоде в контрольной группе у 3 (30\%) животных на 1-е сут после операции была зафиксирована мальпозиция транспедикулярных винтов в позвоночный канал с развитием болевого синдрома, астенизации и невральных расстройств в виде дисфункции тазовых органов. На 90-е сут послеоперационного периода у 1 особи (10\%) был зафиксирован перелом одного из транспедикулярных винтов с нестабильностью конструкции. У животных 


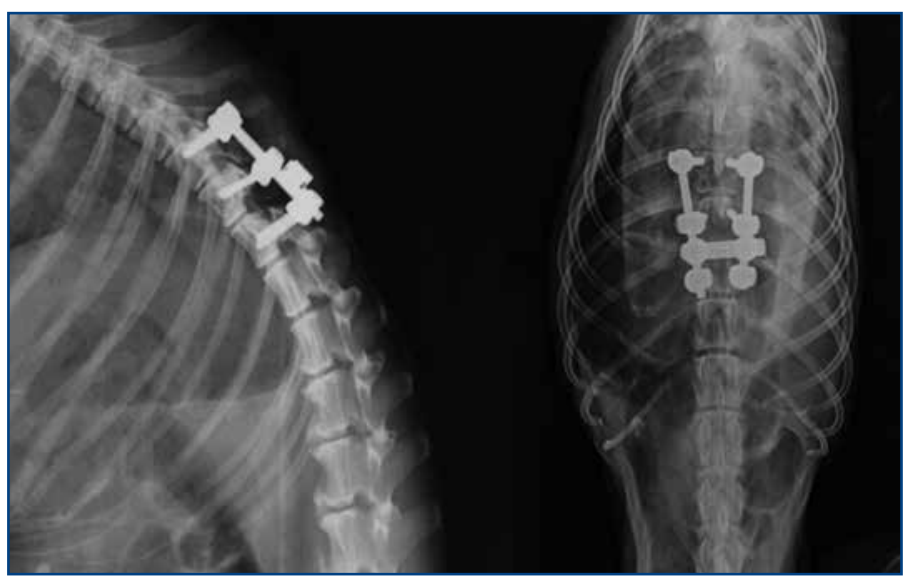

Рис. 3. Рентгенограмма позвоночника животного (особь из контрольной группы), которому проводилась фриксация тел позвонков транспедикулярными винтами

Fig. 3. Spine X-ray imaging (control animal) after vertebral body fixation using transpedicular screws

основной группы, по данным рентгенографии и МСКТ (рис. 4) позвоночного столба в послеоперационном периоде, отсутствовало вхождение винтов в позвоночный канал. По нашему мнению, мальпозиция возникла интраоперационно при установке транспедикулярных винтов.

Оценку неврологического дефицита по шкале Frankel проводили на 90-е сут после операции и сравнивали с исходными показателями (табл. 1). На дооперационном этапе животные в обеих группах в отношении неврологического дефицита по шкале Frankel распределились на уровнях F.A, F.B, F.C практически симметрично. Об этом свидетельствуют данные сравнения по критерию Манна - Уитни (U-статистика 42,0, p-значение 0,26) для независимых наблюдений, позволяющие констатировать, что обе группы принадлежат к одной генеральной совокупности и не имеют статистически значимого сдвига относительно друг друга на дооперационном этапе. В обеих группах в послеоперационном периоде ни у одного из животных не выявлено прогрессирование неврологического дефицита. В контрольной группе на 90-е сут после операции у 2 (20\%) особей достигнут максимальный регресс неврологического дефицита (F.E),

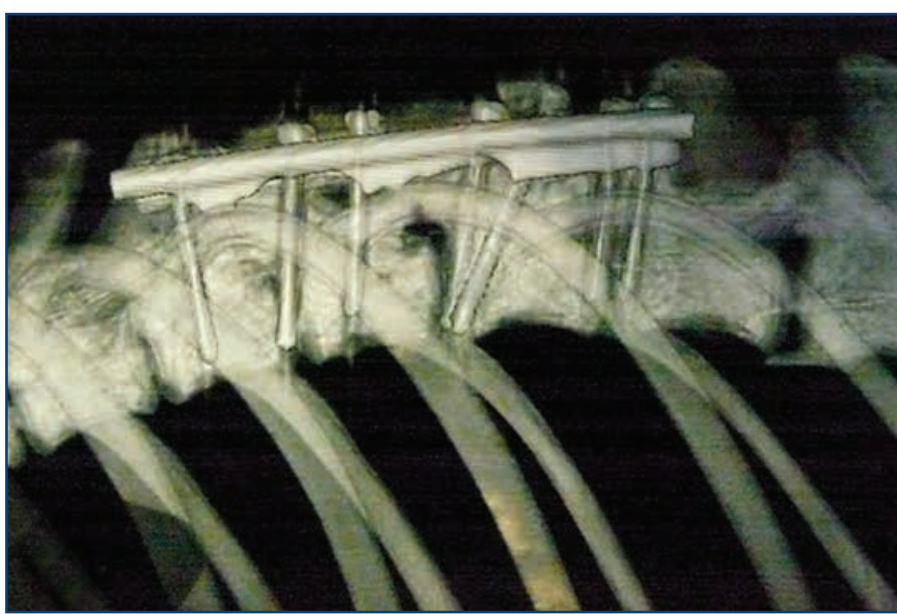

Рис. 4. Данные МСКТ через 90 дней после установки персонифицированной пластины

Fig. 4. Multisliced CT at 90 days after the placement of personalized plate (study animal)

у $5(50 \%)$ - верифицирована F.D степень, в 1 (10\%) и 2 (20\%) случаях выявлены F.В и F.C степени соответственно. Наиболее частым нарушением у животных (3 особи) этой группы были невральные расстройства в виде дисфункции тазовых органов (задержка мочи), требующие длительного послеоперационного выведения мочи разовым жестким уретральным катетером Нелатона. В основной группы на 90-е сут после операции у 2 (20\%) особей достигнут максимальный регресс неврологического дефицита (F.E), у 6 (60\%) - верифицирована F.D степень, в 2 (20\%) случаях установлена F.C степень. Сравнение уровней неврологического дефицита у особей из основной группы на двух этапах выявило статистически значимый (критерий Вилкоксона для зависимых наблюдений $\mathrm{T}=0,0, \mathrm{p}=0,004)$ его регресс в послеоперационном периоде (90-е сут).

\section{ЗАКЛЮЧЕНИЕ}

Фиксация позвоночно-двигательных сегментов с помощью персонифицированной титановой пластины с навигационными отверстиями позволяет избежать

Таблица 1. Характеристика неврологического десрицита по шкале Frankel у животных из контрольной $(\mathrm{n}=10)$ и основной ( $\mathrm{n}=10$ ) групп до и после оперативного вмешательства (к 90-м сут)

Table 1. Neurological deficiency (Frankel scale) in control $(n=10)$ and study $(n=10)$ animals before and after (90 days) the surgery

\begin{tabular}{|c|c|c|c|}
\hline $\begin{array}{c}\text { Неврологический дефицит } \\
\text { по шкале Frankel } \\
\text { Frankel scale for neurological deficiency }\end{array}$ & $\begin{array}{l}\text { Группы } \\
\text { Groups }\end{array}$ & $\begin{array}{c}\text { До оперативного } \\
\text { вмешательства, n (\%) } \\
\text { Before the surgery, n (\%) }\end{array}$ & $\begin{array}{l}\text { 90-е сут после оперативного } \\
\text { вмешательства, } \mathbf{n}(\%) \\
90 \text { days after the surgery, } \mathrm{n}(\%)\end{array}$ \\
\hline F.A & Контрольная /Control & $1(10)$ & 0 \\
\hline F.A & Основная / Study & $2(20)$ & 0 \\
\hline F.B & Контрольная / Control & $6(60)$ & $1(10)$ \\
\hline F.B & Основная / Study & $6(60)$ & 0 \\
\hline F.C & Контрольная / Control & $3(30)$ & $2(20)$ \\
\hline F.C & Основная / Study & $2(20)$ & $2(20)$ \\
\hline F.D & Контрольная / Control & 0 & $5(50)$ \\
\hline F.D & Основная / Study & 0 & $6(60)$ \\
\hline F.E & Контрольная / Control & 0 & $2(20)$ \\
\hline F.E & Основная / Study & 0 & $2(20)$ \\
\hline
\end{tabular}


интраоперационной мальпозиции фиксирующих винтов с развитием болевого синдрома и неврологических расстройств; увеличить стабильность фиксации сегментов позвоночника и профилактировать переломы винтов за счет увеличения площади соприкосновения титановой пластины с костью; проводить фиксацию без применения дорогостоящих и потенциально опасных для пациента и персонала систем электронно-оптической рентгеновской визуализации. Полученные результаты позволяют сделать вывод о возможности и целесообразности применения технологии фиксации позвоночно-двигательных сегментов персонифицированными титановыми пластинами с элементами навигации, произведенными на 3D-принтере с применением аддитивных технологий и с учетом особенностей анатомии, в эксперименте на животных. Элементы разработанной технологии могут быть использованы в практике нейрохирургической клиники после окончания апробации метода в эксперименте.

\section{Благодарность}

Работа выполнена согласно темам НИОКР УНИИФ Минздрава РФ № 114032140001 и ИИФ УрО РАН № 01201352042. Авторы благодарят Центр коллективного пользования ИИФ УрО РАН за оказанную помощь в проведении эксперимента.

\section{Acknowledgements}

The study was performed as a part of Research and Experimental Developmen theme No. 114032140001 of the Ural Research Institute of Phthisiopulmonology - Branch of the Na-tional Medical Research Center of Phthisiopulmonology and Infectious Diseases and theme No. 01201352042 of the Institute of Immunology and Physiology of the Ural Branch of RAS. The authors are grateful to the Center for Collective Use of the Institute of Immunology and Physiology of the Ural Branch of RAS for performing the study.

\section{Лuтература}

1. Хирургическое лечение костно-суставного туберкулеза. Под ред. Ю.Н. Леваше ва, А.Ю. Мушкина. СПб.: Санкт-Петербургский науч.-исслед. ин-т фтизиопульмонологии; 2008

2. Бурлаков С.В., Олейник В.В., Вишневский А.А. Влияние длительности заболева ния туберкулезным спондилитом на развитие осложнений. Травматология и ор топедия России. 2013;1:61-66.

3. Бурлаков С.В., Вишневский А.А. Неэффективное одноэтапное хирургическое лечение распространенного туберкулезного спондилита: клинический случай и обзор литературы. Хирургия позвоночника. 2018;15(1):71-78.

4. Куклин Д.В., Беляков М.В., Сердобинцев М.С., Дорофеев Л.А. Тактика при менения титановых имплантатов при туберкулезном спондилите в зависимо сти от сагиттального профиля позвоночника. Туберкулез и болезни легких. 2015;7:81.

5. Травматология: национальное руководство. Под ред. Г.П. Котельникова С.П. Миронова. М.: ГЭОТАР-Медиа; 2011

6. Перльмуттер О.А. Травма позвоночника и спинного мозга. Н. Новгород: Издательство Нижегородской государственной медицинской академии; 2016.

7. Assaghir Y.M., Refae H.H., Alam-Eddin M. Anterior versus posterior debridement fusion for single-level dorsal tuberculosis: the role of graft-type and level of fixation on determining the outcome. Eur. Spine J. 2016;25:3884-3893. DOI: 10.1007/ s00586-016$4516-2$.

8. Ba Z.Y., Pan F.M., Huang Y.F. et al. One-stage anterior radical debridement and reconstruction with titanium mesh combined with antituberculosis for cervical spinal tuberculosis: 5-13 years follow up. Int. J. Clin. Exp. Med. 2016;9:6368-6372.

9. Liu C., Lin L., Wang W. et al. Long-term outcomes of vertebral column resection for kyphosis in patients with cured spinal tuberculosis: average 8-year follow-up. J. Neurosurg. Spine. 2016;24:777-785. DOI: 10.3171/2015.8.SPINE15534.

10. Kaneyama S., Sugawara T., Sumi M. et al. A novel screw guiding method with a screw guide template system for posterior C2 fixation: clinical article. J. Neurosurg. Spine. 2014;21:231-238. DOI: 10.3171/2014.3.SPINE13730.

11. Liu Z., Wang X.Y., Xu Z.Q. et al. Two approaches for treating upper thoracic spinal tuberculosis with neurological deficits in the elderly: A retrospective case-control study. Clin. Neurolog. Neurosurg. 2016;141:111-116. DOI: 10.1016/j.clineuro.2016.01.002.

12. Ran B., Xie Y.L., Yan L., Cai L. One-stage surgical treatment for thoracic and lumbar spinal tuberculosis by transpedicular fixation, debridement, and combined interbody and posterior fusion via a posterior-only approach. J. Huazhong. Univ. Sci Technolog. Med. Sci. 2016;36:541-547. DOI: 10.1007/s11596-016-1622-7.

13. Sugawara T., Kaneyama S., Higashiyama N. et al. Prospective Multicenter Study of a Multistep Screw Insertion Technique Using Patient-Specific Screw Guide Templates for the Cervical and Thoracic Spine. SPINE;43(23):1685-1694. DOI: 10.1097/ BRS.0000000000002810.

14. Radermacher K., Portheine F., Anton M. et al. Computer assisted orthopaedic surgery with image based individual templates. Clin. Orthop. Relat. Res. 1998;354:28-38.

15. Yang H.D., Hou K.D., Zhang L. et al. Minimally invasive surgery through the interlaminar approach in the treatment of spinal tuberculosis: A retrospective study of 31 patients. J. Clin. Neurosci. 2016;32:9-13. DOI: 10.1016/j.jocn.2015.11.036.

16. Zhang P.H., Peng W., Wang X.Y. et al. Minimum 5-year follow-up outcomes for single-stage transpedicular debridement, posterior instrumentation and fusion in the management of thoracic and thoracolumbar spinal tuberculosis in adults. Br. J.Neurosurg. 2016;30:666-671. DOI: 10.1080/02688697.2016.1206182.

17. Kaneyama S., Sugawara T., Sumi M. et al. A novel screw guiding method with a screw guide template system for posterior C2 fixation: clinical article. J. Neurosurg. Spine. 2014;21:231-238. DOI: 10.3171/2014.3.SPINE13730.

18. Mobbs R.J., Coughlan M., Thompson R. et al. The utility of 3D printing for surgical planning and patient-specific implant design for complex spinal pathologies: case report. J. Neurosurg. Spine. 2017;26:513-518. DOI: 10.3171/2016.9.SPINE16371.

19. Radermacher K., Portheine F., Anton M. et al. Computer assisted orthopaedic surgery with image based individual templates. Clin. Orthop.Relat. Res. 1998;354:28-38.

\section{References}

1. Surgical treatment of osteoarticular tuberculosis / Ed. Yu.N. Levasheva, A.Yu. Mushkina. St. Petersburg: St. Petersburg Scientific Research Institute of Phthisiopulmonology; 2008 (in Russ.)

2. Burlakov S.V., Oleinik V.V., Vishnevsky A.A. The effect of the duration of tuberculous spondylitis on the development of complications. . Travmatologiya i ortopediya Rossii. 2013;1: 61-66.

3. Burlakov S.V., Vishnevsky A.A. Ineffective one-stage surgical treatment of common tuberculous spondylitis: a clinical case and literature review. Khirurgiya pozvonochnika. 2018;15(1):71-78

4. Kuklin D.V., Belyakov M.V., Serdobintsev M.S., Dorofeev L.A. The tactics of using titanium implants for tuberculous spondylitis, depending on the sagittal profile of the spine. Tuberculosis and lung disease. 2015;7:81

5. Traumatology: national guidlines / Ed. G.P. Kotelnikova, S.P. Mironova. M. GEOTAR-Media; 2011.

6. Perlmutter O.A. Injury to the spine and spinal cord. N. Novgorod: Publishing House of the Nizhny Novgorod State Medical Academy; 2016.

7. Assaghir Y.M., Refae H.H., Alam-Eddin M. Anterior versus posterior debridement fusion for single-level dorsal tuberculosis: the role of graft-type and level of fixation on determining the outcome. Eur. Spine J. 2016;25:3884-3893. DOI: 10.1007/ s00586-016$4516-2$.

8. Ba Z.Y., Pan F.M., Huang Y.F. et al. One-stage anterior radical debridement and reconstruction with titanium mesh combined with antituberculosis for cervical spinal tuberculosis: 5-13 years follow up. Int. J. Clin. Exp. Med. 2016;9:6368-6372.

9. Liu C., Lin L., Wang W. et al. Long-term outcomes of vertebral column resection for kyphosis in patients with cured spinal tuberculosis: average 8-year follow-up J. Neurosurg. Spine. 2016;24:777-785. DOI: 10.3171/2015.8.SPINE15534.

10. Kaneyama S., Sugawara T., Sumi M. et al. A novel screw guiding method with a screw guide template system for posterior C2 fixation: clinical article. J. Neurosurg. Spine. 2014;21:231-238. DOI: 10.3171/2014.3.SPINE13730.

11. Liu Z., Wang X.Y., Xu Z.Q. et al. Two approaches for treating upper thoracic spina tuberculosis with neurological deficits in the elderly: A retrospective case-control study. Clin. Neurolog. Neurosurg. 2016;141:111-116. DOI: 10.1016/j.clineuro.2016.01.002. 12. Ran B., Xie Y.L., Yan L., Cai L. One-stage surgical treatment for thoracic and lumbar spinal tuberculosis by transpedicular fixation, debridement, and combined interbody and posterior fusion via a posterior-only approach. J. Huazhong. Univ. Sci Technolog. Med. Sci. 2016;36:541-547. DOI: 10.1007/s11596-016-1622-7.

13. Sugawara T., Kaneyama S., Higashiyama N. et al. Prospective Multicenter Study of a Multistep Screw Insertion Technique Using Patient-Specific Screw Guide Templates for the Cervical and Thoracic Spine. SPINE;43(23):1685-1694. DOI: 10.1097/ BRS.0000000000002810.

14. Radermacher K., Portheine F., Anton M. et al. Computer assisted orthopaedic surgery with image based individual templates. Clin. Orthop. Relat. Res. 1998;354:28-38.

15. Yang H.D., Hou K.D., Zhang L. et al. Minimally invasive surgery through the interlaminar approach in the treatment of spinal tuberculosis: A retrospective study of 31 patients. J. Clin. Neurosci. 2016;32:9-13. DOI: 10.1016/j.jocn.2015.11.036.

16. Zhang P.H., Peng W., Wang X.Y. et al. Minimum 5-year follow-up outcomes for single-stage transpedicular debridement, posterior instrumentation and fusion in the management of thoracic and thoracolumbar spinal tuberculosis in adults. Br J.Neurosurg. 2016;30:666-671. DOI: 10.1080/02688697.2016.1206182.

17. Kaneyama S., Sugawara T., Sumi M. et al. A novel screw guiding method with a screw guide template system for posterior C2 fixation: clinical article. J. Neurosurg. Spine. 2014;21:231-238. DOI: 10.3171/2014.3.SPINE13730.

18. Mobbs R.J., Coughlan M., Thompson R. et al. The utility of 3D printing for surgica planning and patient-specific implant design for complex spinal pathologies: case report. J. Neurosurg. Spine. 2017;26:513-518. DOI: 10.3171/2016.9.SPINE16371.

19. Radermacher K., Portheine F., Anton M. et al. Computer assisted orthopaedic surgery with image based individual templates. Clin. Orthop. Relat. Res. 1998;354:28-38. 


\section{Сведения об авторах:}

${ }^{1}$ Доценко Иван Александрович - научный сотрудник, врач-нейрохирург, ORCID iD 0000-0002-1897-5274;

${ }^{2}$ Котомиев Вячеслав Владимирович - д.м.н., профессор, ведущий научный сотрудник лаборатории иммунофизиологии и иммунофармакологии ORCID iD 0000-0001-52329244;

${ }_{1,3}$ Медвинский Игорь Давыдович- д.м.н., профессор, руководитель отдела, профессор кафедры фтизиопульмонологии, ORCID iD 0000-0002-3064-4865;

${ }^{1}$ Савельев Александр Владимирович - к.м.н., старший научный сотрудник, заведующий отделением лучевой диагностики, ORCID iD 0000-0001-8100-625X

${ }^{4}$ Ботов Михаил Алексеевич - старший преподаватель кафедры математики ORCID iD 0000-0002-1157-6895.

${ }^{1}$ УНИИФ - филиал ФГБУ «НМИЦ ФПИ» Минздрава России. 620039, Россия, г. Екатеринбург, ул. 22-го партсъезда, д. 50. ${ }^{2}$ ИИФ УрО РАН, 620049, Россия, г. Екатеринбург, ул. Первомайская, д. 106.

${ }^{3}$ ФГОУ ВО УГМУ Минздрава России. 620028, Россия, г. Екатеринбург, ул. Репина, д. 3.

${ }^{4}$ ФАОУ ВО «УрФУ имени первого президента России Б.Н. Ельцина». 620002, Россия, г. Екатеринбург, ул. Мupa, д. 19.

Контактная информация: Медвинский Игорь Давыдович, e-mail: 2134012@gmail.com. Прозрачность финансовой деятельности: авторы не имеют финансовой заинтересованности в представленных материалах или методах. Конфликт интересов отсутствует. Статья поступила 26.02.2020.
About the authors:

${ }^{1}$ Ivan A. Dotsenko - MD, researcher, neurosurgeon, ORCID iD 0000-0002-1897-5274;

${ }^{2}$ Vyacheslav V. Kotomtsev - MD, PhD, Professor, leading researcher of the Laboratory of Immunophysiology and Immunopharmacology ORCID iD 0000-0001-5232-9244;

1,3 Igor Medvinskiy - MD, PhD, Professor, Head of the Division, professor of the Department of Phthisiopulmonology, ORCID iD 0000-0002-3064-4865;

${ }^{1}$ Aleksandr V. Savel'ev - MD, PhD, senior researcher, Head of the Department of Diagnostic Radiology, ORCID iD 0000 0001-8100-625X;

${ }^{4}$ Mikhail A. Botov - senior lecturer of the Department of Mathematics ORCID iD 0000-0002-1157-6895.

${ }^{1}$ Ural Research Institute of Phthisiopulmonology - Branch of the National Medical Research Center of Phthisiopulmonology and Infectious Diseases. 50, 22 ${ }^{\text {nd }}$ Parts'ezd str., Ekaterinburg, 620039, Russian Federation.

${ }^{2}$ Institute of Immunology and Physiology of the Ural Branch of the Russian Academy of Sciences. 106, Pervomaiskaya str., Ekaterinburg, 620049, Russian Federation.

${ }^{3}$ Ural State Medical University. 3, Repin str., Ekaterinburg, 620028, Russian Federation.

${ }^{4}$ The First Russian President B.N. Yeltsin Ural Federal University. 19, Mira str., Ekaterinburg, 620002, Russian Federation.

Contact information: Igor Medvinskiy, e-mail: 2134012@ gmail.com. Financial Disclosure: no authors have a financial or property interest in any material or method mentioned. There is no conflict of interests. Received 26.02.2020. 